\title{
ENERGY-BALANCED IMPROVED LEACH ROUTING PROTOCOL FOR WIRELESS SENSOR NETWORKS
}

\author{
KHAMISS.A.A, CHAI Senchun, ZHANG Baihai and LI Qiao \\ School of Automation, Beijing Institute of Technology, Beijing, China \\ Ahmed.abdo7728@yahoo.com, Chaisc970bit.edu.cn, \\ smczhanglbit.edu.cn, 442302839 @qq. com
}

\begin{abstract}
A proper sensor node clustering is an effective topology control that can balance energy consumption among sensor nodes and increase network scalability and life time. As the use of wireless sensor networks (WSNs) has grown enormously, the need for energy-efficient routing and data aggregation has also risen. LEACH (Low Energy Adaptive Cluster Hierarchy) is a hierarchical clustering protocol that provides an elegant solution for such protocols. Random clustering is the main deficiency of LEACH. In this paper an energy balanced clustering approach is proposed, in which the K-mean clustering algorithm is applied. It is centralized clustering algorithm that based on minimum energy clustering to form optimal clusters. For the candidate nodes, the location and the residual energy are used as key parameters to select the cluster head $(\mathrm{CH})$. The method shows that the proposed approach outperforms LEACH in terms of energy conservation and network life time prolonging.
\end{abstract}

\section{KEYWORDS}

K-mean, Role Factor, Cluster Energy \& Root Node.

\section{INTRODUCTION}

WSNs typically consist of a large number of low-cost, low-power and multifunctional wireless sensor nodes, with sensing, limited communication and computation capabilities. These nodes communicate via a wireless medium and collaborate to accomplish a common task, such as environment monitoring, military surveillance and industrial process control [1]. The basic philosophy behind WSNs is that, while the capability of each individual node is limited, the aggregate power of the entire network is sufficient for the required mission [2]. In most applications, nodes are deployed randomly, and once deployed they must be able to autonomously organize themselves into a network. Generally WSNs are characterized with high density of deployed nodes, while the nodes themselves are unreliable and restricted in power, computation and storage resources. And due to these characteristics, suitable network routing protocols are required to implement various network control and management functions. A WSN typically has little or no infrastructure; and a great number of nodes allow sensing larger geographical region with greater accuracy. The collected data is sent usually via radio transmitter to the sink either directly or through a gate way. Routing protocol is one of the core technologies in WSNs that is full of challenge due to its inherent characteristics [3, 4]. Routing is the process of determining the best data transmission path between source and destination. Many protocols

Natarajan Meghanathan et al. (Eds) : WiMONe, NCS, SPM, CSEIT - 2014

pp. 01-11, 2014. (C) CS \& IT-CSCP 2014

DOI : $10.5121 /$ csit.2014.41201 
have been proposed to forward the correct data to the sink and enhance the life time of the WSN [5]. Using clustering techniques in routing protocols prolongs the life time of the network and contributes in over all system scalability. Clustering is a well-known and widely used data analysis technique that is useful in applications which require scalability. Data transmission is the most critical energy consumption issue for sensor node, so, by clustering, communication can be single-hop or multi-hop. A single-hop communication can reduce the over head cost, but when communication distance increases, it consumes more energy. Multi-hop communication consumes less energy than single-hop for long distances, but it has more over head cost and delay. Then, multi-hop routing is suitable for applications with large scale networks. The routing protocols in WSNs can be classified into two categories according to application requirements, namely data-query based routing and data-gathering based routing [6]. The Energy-efficient routing forwards packets along the path with minimum energy, this leads to minimum energy consumption. But it causes unbalanced distribution of residual energy and nodes closer to sink deplete their energy faster than others. Such imbalance definitely shortens the life time of the network. And the connectivity between nodes and sink can be maintained for longer time if nodes consume their energy evenly. Therefore, balanced energy consumption must be considered beside energy-awareness when designing energy-efficient routing protocol. The energy balanced routing distributes the levels of residue energy evenly throughout the network, extending the network life time. Recently, many research activities have been done in designing energy-efficient protocols for WSNs. Most of them focus on finding minimum energy path for data transmission and neglect the survivability of the entire network. Minimum energy consumption schemes minimize energy consumption by forwarding data through few popular paths. Therefore, energy consumption on these paths increases quickly causing imbalance of residual energy throughout the network, decreasing the overall performance and life time of the network [7].

Generally, the main reasons that cause imbalance of energy distribution in WSNs can be summarized in:

- Topology: The initial deployment limits the number of paths through which data can flow.

- Application: The applications may determine the location and the rate at which the nodes generate data. So, the area generating more data and the path forwarding more data may deplete energy faster.

- Routing: Minimizing energy consumption via using static optimal path results in energy imbalance because energy of optimal path nodes is quickly depleted.

According to the above reasons, some possible solutions had been suggested to balance energy consumption:

- Deployment optimization: This will solve the problem of mismatch between topology and application according to the traffic pattern.

- Topology control: Nodes collaboratively adjust their transmission power to form a proper network topology.

- Relay nodes: Relay nodes can relieve heavily loaded areas or paths in a way similar to optimization of deployment.

- Data aggregation: It exploits redundancy to minimize energy consumed in data transmission.

- Energy-Balanced Routing: It maintains the network connectivity for a longer time and prolongs the entire network life time [8].

WSNs are resource constrained, so, such networks need novel protocols and algorithms which can utilize the available resources optimally and meet user requirements. The above points, in 
addition to energy-efficiency and balanced energy consumption are important issues when designing energy-efficient routing protocols for WSNs. In this paper we proposed a routing scheme that overcomes the problem of energy imbalance in LEACH routing protocol. It demonstrates the advantages of balanced energy consumption across the network. The remainder of this paper is organized as follows: The next section describes the related works and section III explains the system model. Section IV demonstrates the proposed approach while section V contains the results obtained; and finally section VI represents the conclusion of the work.

\section{RELATED WORKS}

Routing in WSNs is very challenging due to the inherent characteristics that distinguish the WSNs from other wireless networks like ad hoc or cellular networks. The aim of network layer is to route data from sensor to sink in an energy-efficient and reliable manner in order to extend the network life time. Many research activities had addressed the energy-efficient routings; most of them focused on minimizing energy consumption on local nodes or the entire network via finding an optimal path. In [2], some modified versions of LEACH had been discussed. LEACH is a cluster-based protocol that randomly selects $\mathrm{CHs}$, where the number of $\mathrm{CHs}$ is adaptive and the selection process is dynamically rotated to evenly distribute load [9]. LEACH suffers from random selection of $\mathrm{CHs}$ and it works best only if the energy of nodes is uniform. There is no any certainty about the distribution of $\mathrm{CHs}$ throughout the network, and the idea of dynamic clustering brings extra overhead. Moreover, it is not applicable for large scale applications. ELEACH, improves the $\mathrm{CH}$ selection by making the residual energy of the node as the main metric which decides whether the nodes turn into $\mathrm{CHs}$ or not after the first round. In the first round all nodes have the same probability to become $\mathrm{CH}$, i.e. randomly as $\mathrm{LEACH}$, in the next rounds the residual energy of each node is different and it is taken into account. That means, nodes have more energy will become CHs rather than nodes with less energy [10]. TL-LEACH (Two-level Hierarchy LEACH) is enhanced version of LEACH protocol; it has two levels of CHs. In this protocol, $\mathrm{CH}$ collects data from other cluster members as original $\mathrm{LEACH}$, but rather than transfer data to the BS directly, it uses one of the $\mathrm{CHs}$ that lies between the $\mathrm{CH}$ and the BS as a relay station [11].TL-LEACH faces the same problem as LEACH, since it uses the same mechanism. LEACH-C is a centralized clustering algorithm; it produces better clusters by dispersing the CHs evenly through the network. During the setup phase of LEACH-C each node sends information about its current location and residual energy to the sink. Sink node computes the node average energy and determines which nodes have energy below this average [9]. In $\mathrm{LEACH}$, each $\mathrm{CH}$ directly communicates with the BS no matter the distance between $\mathrm{CH}$ and BS. On the other hand M-LEACH (multi-hop LEACH) selects the optimal path between the $\mathrm{CH}$ and BS through other $\mathrm{CHs}$ and uses the $\mathrm{CHs}$ as a relay station [12]. M-LEACH is almost the same as LEACH, only makes communication mode between CHs and BS from single hop to multi hop. In LEACH-H (Hybrid cluster head selection LEACH) the base station selects the CHs in first round. While in the followed rounds the CHs select the new CHs in their own clusters. Hybrid EnergyEfficient Distributed Clustering (HEED) is a multi hop clustering that focus on efficient clustering based on the physical distance between nodes. The scheme selects $\mathrm{CHs}$ in terms of residual energy and intra-cluster communication cost, which is useful if given node falls within the range of more than $\mathrm{CH}$ [13]. The problem in HEED is that, it is important to identify what is the range of a node in terms of its power level, as node will has multiple discrete transmission power levels. In [14], FZ-LEACH (Far-Zone LEACH) is proposed to form far-zone which is a group of nodes which are placed at locations where their energies are less than a threshold. In ACHTH- LEACH (Adaptive Cluster Head Election and Two-Hop LEACH) protocol [15], nodes are tagged as near or far nodes according to the distances to the BS. The near nodes belong to one cluster, while the far nodes are divided in to different clusters. The node with the maximal residual energy in each cluster is selected as $\mathrm{CH}$. 
In this paper we propose scheme that provides how to prolong the network life time and make an efficient use of the critical resources located at the nodes. We aim to create more intelligent clusters, minimize the number of nodes in a cluster, and select only a single $\mathrm{CH}$ in each cluster and not to affect the communication functionalities. The scheme adopted minimum energy clustering technique to form clusters and minimum communication cost metric to select $\mathrm{CH}$ in each cluster. Also minimum energy path multi-hop routing had been used to transmit data to the base station.

\section{SENSOR RADIO MODEL}

\subsection{Assumptions}

The following assumptions are made about the sensors and the network model:

1-The sink node is located inside the sensing field.

2-Sensors are location-aware; energy constrained

3-Sensors are energy-aware; energy resource-adaptive.

4-Links are symmetric; nodes and sink both are stationary

5-Cluster: a mixture of single and multi-hop, no assumption about homogeneity of node category and homogeneity of dispersion radius.

\subsection{Sensor Energy Model}

We adopt he energy consumption and radio model given in [9]. To achieve an acceptable signalto-noise ratio (SNR) in transmitting $k$ bit message over a distance $d$, the energy cost of transmission $\left(E_{T X}\right)$ is given by:

$$
\left\{\begin{array}{l}
E_{T X}(k, d)=k E_{\text {elec }}+k \varepsilon_{f s} d^{2} ; \text { if } \mathrm{d}<=d_{0} \\
E_{T X}(k, d)=k E_{\text {elec }}+k \varepsilon_{m p} d^{4} ; \text { if } \mathrm{d}>d_{0}
\end{array}\right.
$$

Where $E_{\text {elec }}=50$ nj is the energy utilized to run the transceiver circuit, $\mathcal{E}_{f s}=10 \mathrm{j} / \mathrm{bit} / \mathrm{m}^{2}$ and $\varepsilon_{m p}=0.0013 \mathrm{pj} / \mathrm{bit} / \mathrm{m}^{4}$ are energies utilized by transmission amplifiers for short and long distances respectively. For $d=d_{0}$, the distance threshold is given by, $d_{0}=\sqrt{\frac{\varepsilon_{f s}}{\varepsilon_{m p}}}$ where $d_{0}$ is known as the crossover distance and $E_{d}=5 \mathrm{nj} /$ bit / signal is the fusion energy per bit. To receive a $k$ bit message, the radio expends receiving energy ( $E_{R X}$ ) which is given by:

$$
E_{R X}=k E_{\text {elec }}
$$

\section{PROPOSED ALGORITHM}

The proposed scheme is a cluster-based algorithm that modifies the clustering process. Thus, both the cluster formation and the $\mathrm{CH}$ selection methods had been modified. For more energy conservation, a multi-hop routing was adopted to forward data to the BS. Clustering achieves an important improvement in terms of energy consumption, and it is crucial in scaling the networks. 
Also clustering is one of the basic approaches to design energy-efficient distributed WSNs. However, these benefits can result in extra overhead due to cluster formation's message exchange. In this scheme we assumed exchanging sensor data may be an expensive network operation, but exchanging data about sensor data needs not be. Clustering can be performed in centralized way by BS (as in our scheme) or in distributed way where every node decides autonomously about its role. Furthermore, cluster formation can be either static or dynamic according to whether the network is heterogeneous or homogenous. Since energy is the major concern, then balanced energy consumption is important in energy conservation. The operation of the proposed scheme is divided into rounds; each round consists of setup phase and data transmission phase. The cluster formation, $\mathrm{CHs}$ selection, and multi-hop paths establishment are done successively in setup phase. While in data transmission phase, the nodes sense and transmit data to $\mathrm{CHs}$ and then $\mathrm{CHs}$ forward data to $\mathrm{BS}$ after aggregation. In $\mathrm{LEACH}$, the cluster heads are selected according to formula (3) and then members joint their nearest $\mathrm{CHs}$ to construct clusters in setup phase. In the proposed scheme, clusters are formed firstly, and then a single cluster head is selected in each cluster.

$$
T(n)=\left\{\begin{array}{cc}
\frac{p}{1-p^{*}\left(r \bmod \left(\frac{1}{p}\right)\right)} \quad \text { if } n \in G \\
0 \quad \text { if } n \notin G
\end{array}\right.
$$

Where $p$ represents the percentage of $\mathrm{CHs}$ in the network, $r$ is the current round and $G$ represents the group of nodes those had not been selected as cluster head.

\subsection{Setup Phase}

\subsubsection{Cluster Formation}

Firstly, since the BS resources are not limited, the BS uses the unsupervised clustering technique $\mathrm{K}$-mean Clustering method to divide the nodes into k clusters $(\mathrm{k}=p \times n)$. It can be viewed as a greedy algorithm for partitioning the total $n$ nodes into $\mathrm{k}$ clusters so as to minimize the sum of squared distances to the cluster centers. The division based on network topology, nodes distribution and the similarity of criterion for grouping. For K-mean clustering method, the similarity criterion in our case is the geometrical distance. Each node is randomly assigned to a cluster, and then the cluster centers are computed. Repeating of this process produces clusters with minimum energy. Cluster energy is defined as the sum of the distance squared from each node to its cluster center. It is the goal of the $\mathrm{K}$ - mean algorithm to find, for fixed number of clusters, a clustering that minimizes this energy. The algorithm aims at minimizing an objective function, which is the squared error function;

$$
J=\sum_{j=1}^{k} \sum_{i=1}^{n}\left\|x_{i}^{j}-c_{j}\right\|^{2}
$$

Where $\left\|x_{i}^{j}-c_{j}\right\|^{2}$ represents chosen distance measure between a node $x_{i}^{j}$ (node $i$ in cluster $j$ ) and cluster center $c_{j}$ (center of cluster $j$ ), $J$ is an indicator of the distance of the total $n$ nodes from their respective cluster centers. The algorithm works as follows; in the beginning, determine the number of clusters $\mathrm{k}$, the centers of these clusters (initial) might be assumed 
randomly or the first $\mathrm{k}$ nodes can serve as the initial centers. K-mean algorithm will do the following three steps until converge (iterate until stable, i.e. no object move group):

1- Determine the centroids coordinate.

2- Determine the distance of each node to the centroids.

3- Group the nodes based on minimum distance.

Fig.1 summarizes the steps of k-mean. After partitioning, the BS notifies the nodes by sending a message containing the cluster ID, distance to cluster centroid, distance to BS and TDMA schedules for each cluster to be employed in steady state phase. Every node stores this message and uses its contents to decide whether it will turn into cluster head or not.

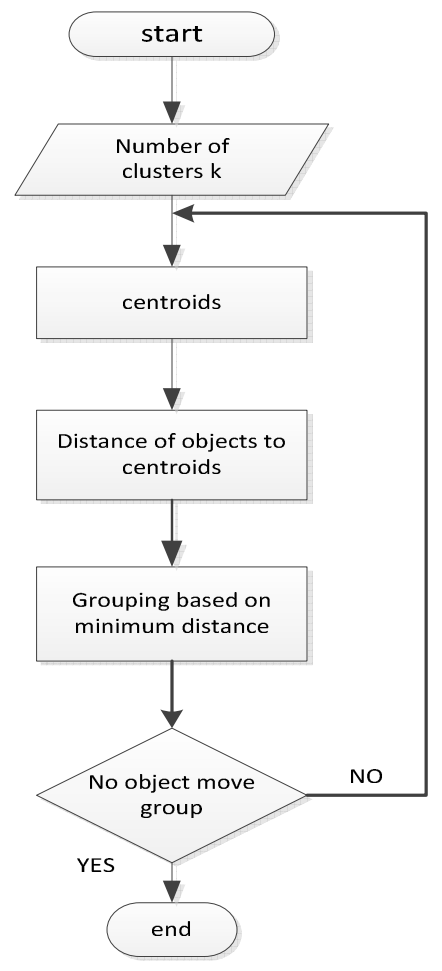

Fig 1. K-mean algorithm steps

\subsubsection{Cluster Head Selection}

Cluster Head Selection: In LEACH, CH selection based on the remaining energy, percentage of $\mathrm{CHs}$ in the network and the number of times the node has been $\mathrm{CH}$. In the proposed algorithm, the $\mathrm{CH}$ selection equation of LEACH had been extended by inserting the residual energy and the distances to BS and cluster centroid. Thus, a node with more residual energy and close to both cluster center and $\mathrm{BS}$ will be more eligible to become a $\mathrm{CH}$ in each cluster. $\mathrm{CH}$ selection equation in (3) can be rewritten as:

$$
T(n)=\frac{p}{1-p *\left(r \bmod \left(\frac{1}{p}\right)\right)} * \frac{E_{\text {resi }}}{E_{\text {initial }}} * \frac{1}{\left(d_{B S}{ }^{2}+{d_{\text {Cent }}}^{2}\right)}
$$


Where $E_{\text {resi }}$ represents the residual energy of node $n, E_{\text {initial }}$ stands for initial energy of all nodes (assuming that all nodes have equal initial energy), and $d_{B S}$ and $d_{C e n t}$ are the distances of node $n$ to the BS and cluster center respectively. In the proposed algorithm, the probability of being $\mathrm{CH}$ increases when a node has more residual energy and more closer to the BS and cluster centroid. Such situation gives a good chance to random number $r$ chosen by the concerned node $n$ to be less than the threshold value $T(n)$. Hence, in each group of nodes, those have the same centroid; the node that has the minimum value of $\mathrm{r}$ becomes a $\mathrm{CH}$ in that group. The $\mathrm{CHs}$ advertise themselves and their centroids, and then every member node belongs to its $\mathrm{CH}$ according to the cluster centroid and receives its TDMA schedule. The selected $\mathrm{CH}$ will continue to work as cluster head until it dies or another $\mathrm{CH}$ dies.

\subsubsection{Establishing Multi Hop Paths}

The proposed algorithm takes a mixture of single and multi-hop routing. At first, the BS computes and broadcasts the average distance $d_{\text {avg }}$ to all nodes according to $d_{\text {avg }}=\frac{\sum_{i=1}^{N} d_{B S}}{N}$. If the distance between $\mathrm{CH}$ and $\mathrm{BS}$ is less than or equal to the average distance the $\mathrm{CH}$ communicates with BS directly. Otherwise, $\mathrm{CH}$ sends a message to other neighbor $\mathrm{CHs}$ including cluster head ID, distance to BS and residual energy. Neighbor CHs save this message at first, then feedback their own messages. Based on the feedback, the $\mathrm{CH}$ chooses a neighbor $\mathrm{CH}$ which has more residual energy and close to BS as its next hop node. Finally, all CHs find their parent nodes, and the parent node that directly communicates with BS is called root node. The root node sends data to BS directly without aggregation.

\subsection{Data Transmission Phase}

This phase consists of frames; in each frame all nodes send data to their CHs or BS. The CHs open their radios to receive data from nodes. Normal nodes are placed in to sleep mode, and every one opens its radio in its own time-slot. Every node saves its residual energy when it is sending data to $\mathrm{CH}$ or to the BS. When any cluster head dies, the BS informs the network nodes about the starting of the cluster reformation step. Then the data transmission phase is terminated and the entire system moves to the setup phase. Because when some nodes die, the total number of nodes decreases and the distribution of the nodes changes, hence, the number of cluster heads must be adjusted to keep the adaptivity of the algorithm. The system continues these rounds until every node's energy has been depleted.

\subsection{Energy Analysis}

Assuming ${ }_{N}$ is the total number of nodes and $K$ is the number of clusters; the average number of nodes per cluster is $\frac{N}{K}$. Each $\mathrm{CH}$ dissipates energy of receiving data from members, aggregating and transmitting the aggregated data to the BS. So, the energy dissipated by the $\mathrm{CH}$ during single frame is:

$$
E_{C H}=l E_{\text {elec }}\left(\frac{N}{K}-1\right)+l E_{D A}\left(\frac{N}{K}\right)+E_{\text {elec }}\left(c \cdot \frac{N}{K} \cdot l\right)+\varepsilon_{m p}\left(c \cdot \frac{N}{K} . l\right) d_{B s}^{4}
$$


$E_{C H}$ represents the total energy dissipated by $\mathrm{CH}$ during each frame, $l E_{\text {elec }}\left(\frac{N}{K}-1\right)$ is the energy dissipated for receiving data from members, $l E_{D A}\left(\frac{N}{K}\right)$ is the aggregation energy and the transmitting energy to the $\mathrm{BS}$ is $E_{\text {elec }}\left(C \cdot \frac{N}{K} \cdot l\right)+\varepsilon_{m p}\left(C \cdot \frac{N}{K} \cdot l\right) * d_{B s}^{4} ; C$ is the aggregation coefficient and $l$ is the message length. On the other hand, member nodes need energy to transmit data to $\mathrm{CH}$ once during each frame, which can be estimated as:

$$
E_{\text {mem }}=l E_{\text {elec }}+l E_{f s} * d_{C H}^{2}
$$

Where $d_{C H}^{2}$ is the distance between $\mathrm{CH}$ and member node in the same cluster. Therefore, the energy dissipated in a cluster during each frame is

$$
E_{\text {cluster }}=E_{C H}+\left(\frac{N}{K}-1\right) E_{m e m}
$$

And the total energy for one frame is:

$$
E_{\text {total }}=K E_{\text {cluster }}
$$

Then according to the number of frames in each round, the life time of the network can be calculated in terms of number of rounds. Number of rounds can be calculated by dividing the total network energy by energy expended in one round.

\section{SIMULATION AND RESULTS}

For simulation environment, 100 nodes are deployed randomly over an area of $100 \times 100$ meters, and the BS is located in the sensing field. The control and data message lengths are 200bits and 6400 bits respectively, with aggregation factor $c$ set to unity as in (6), such that every node has data to send in each round. In this work the following metrics are used to evaluate the life time and performance of the network; time taken till all nodes die, data sent to BS and stability period which is the time till the first node die. Fig. 2 shows the live nodes versus simulation time, from the figure, in the proposed approach the number of rounds during simulation time is nearly twice the number of rounds in normal LEACH and the stability period is increased by $29 \%$. This demonstrates the dramatic improvement in the network life time and performance. 


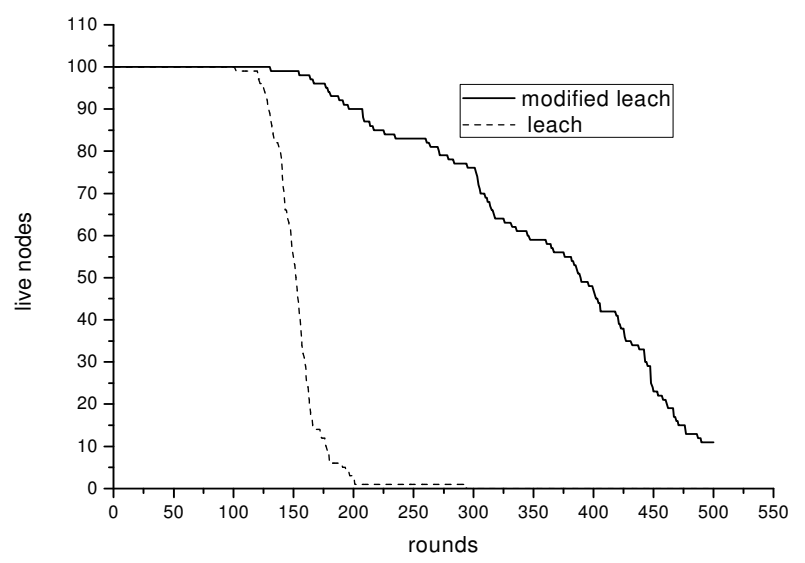

Fig 2. Live nodes over simulation time

This is due to the minimum energy clustering technique and the selection of the most suitable nodes as CHs. The two techniques minimize the cost in terms of energy while nodes are communicating with $\mathrm{CHs}$ or the later are communicating with BS. In fact the mission of each node depends on a factor that consists of the node energy, the minimum energy needed for that mission and the location of the node. This permits nodes to dissipate energy evenly, which lets nodes to stay alive for long time. Fig. 3 shows the data sent to BS during the simulation, the data sent to BS during the simulation time is increased by approximately $36 \%$ than normal LEACH. This is due to the fact that the network life time is increased in compare to LEACH, which demonstrates the significant improvement in the network performance with the proposed approach.

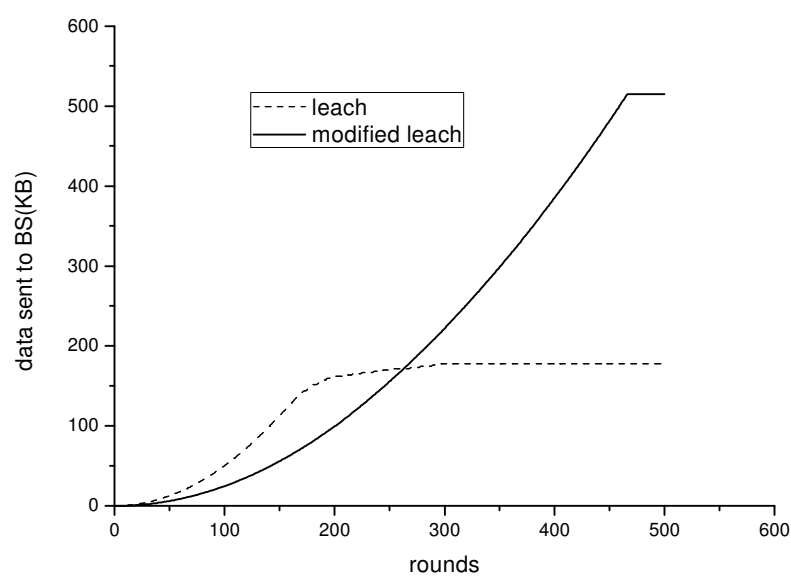

Fig 3. Data sent to BS over simulation time

Fig. 4 and Fig. 5 show the energy variation versus data sent and time respectively. The mission of each node depends on the role factor, so the heavy load mission will be done by nodes that have high role factor, while nodes with low role factor do sensing mission. This explains why the system stays stable for long time. Thus, in proposed approach the network does not exhaust energy suddenly and quickly. From the above results the minimum cluster energy method prolongs the stability period and life time of the wireless network, there by improves the efficiency of the network. 


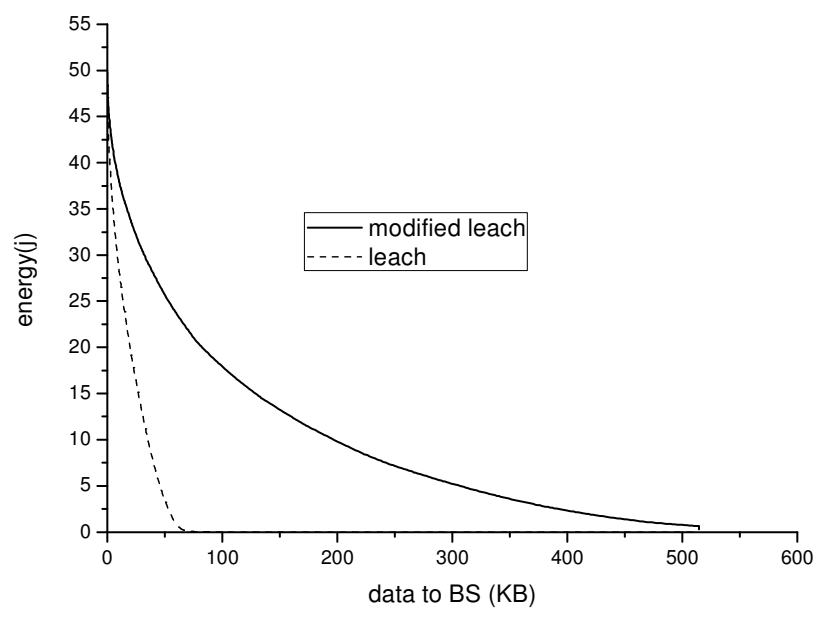

Fig 4. Energy over data sent to BS

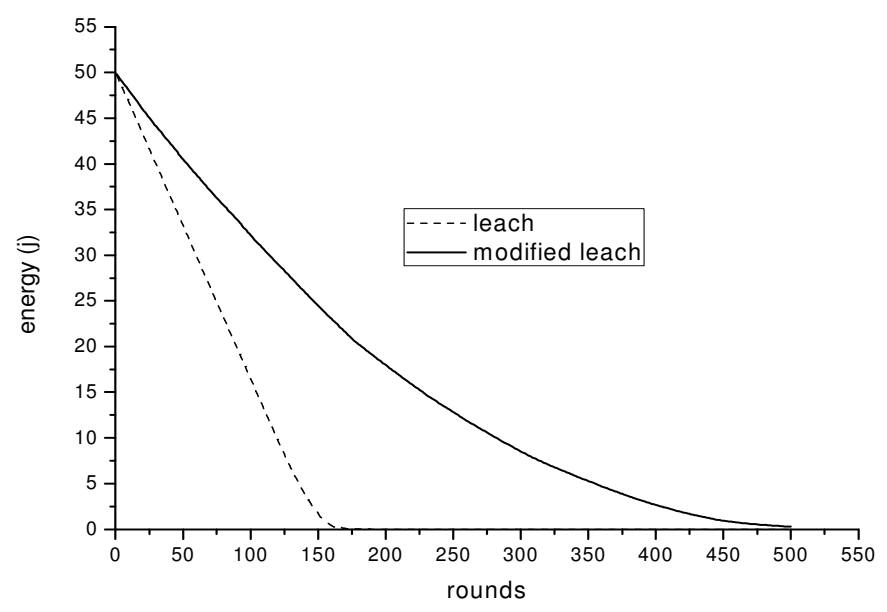

Fig 5. Energy over rounds

\section{CONCLUSIONS}

The transmission distance has a great impact on the energy consumption as well as the regular consumption of the energy over the entire network. Most energy-efficient protocols focus on minimum energy path, although balanced energy consumption has direct effect on energy efficiency. So, a technique that minimizes the cluster energy was used to form minimum energy clusters, in addition to energy-aware method to select the $\mathrm{CH}$. Nevertheless, a minimum energy path is used to forward data in multi-hop routing to minimize energy consumption. The simulation results show that the algorithm can balance the load between nodes and prolong the stability period and life time of the network in comparison to LEACH. The approach does not require cluster formation in every round, but it depends on current $\mathrm{CH}$ state, this decreases the dynamic clustering overhead. Although the results confirmed that the proposed algorithm outperforms LEACH in lengthening WSN life time, there are many protocols that have to be 
compared. Furthermore, the number of member nodes in clusters, the duration of the data transmission phase and whether k-mean will always terminate quickly and its complexity will be studied in future. And more factors that can affect the life time of WSN will be considered. It can be proved that K-mean will always terminate, but the algorithm does not necessary find the most optimal configuration, corresponding to the global objective function minimum. Also it is significantly sensitive to the initial randomly selected cluster centers, since it is iterative clustering algorithm. So, a "good" selection of initial cluster centers is an essential clustering problem.

\section{REFERENCES}

[1] K. Akkaya and M.Younis, (2005), "Asurvey of Routing Protocols in Wireless Sensor Networks", Elsevier Ad Hoc Network Journal, Vol. 3/3, pp. 325-349.

[2] C. Shilpa, Dr. M. Sona and C. Yogesh, (July 2012)"Asurvey of Hierarchical Routing Protocols in Wireless Sensor Networks", International Journal of Emerging trends in Engineering and Development, ISSN 2249-6194, Issue 2, Vol. 5

[3] Jennifer Yick, Biswanath Mukherjee, Dipak Ghosal,Wireless sensor network survey Department of Computer Science, University of California, Davis, CA 95616, United States

[4] H. W. Kim, H. S. Seo, (2010) "Modeling of Energy-efficient Applicable Routing Algorithm in WSN", International Journal of Digital Content Technology and its Applications, vol. 4, no. 5,pp.1322.

[5] S.Taruna, Rekha Kumawat, G.N.Purohit , (August 2012) "Multi-Hop Clustering Protocol using Gate wayNodes in Wireless Sensor Networks", International Journal of Wireless \&Mobile Networks (IJWMN) VOL.4, No.4.

[6] K. Ramesh and Dr. K. Somasundaram,(November 2011) "Acomparative Study of Cluster head Selection Algorithms in Wireless Sensor Networks", International Journal of Computer Science \&Engineering Survey(IJCSES), VOL.2, No.4.

[7] S. Olariu and I. Stojmenovi_c , (2006) "Design Guidelines for MaximizingLifetime and Avoiding Energy Holes in Sensor Networks withUniform Distribution and Uniform Reporting,", Proc. IEEEINFOCOM.

[8] Fengyuan Ren, Jiao Zhang, Tao He, CXhuang Lin and Sajal K. Das, (December 2011)" EBRP: Energy Balanced Routing Protocol for Data gathering in WSNs", IEEE Transactions on parallel and distributed systems, VOL.22, No.12.

[9] W. Heinzelman, A. Chandrakasan, and H. Balakrishnan, (2002) "Anapplication-specific protocol architecture for wirelessmicrosensor networks," IEEE Transaction on WirelessCommunications, , vol. 1 , no. 4, pp. 660-670.

[10] Fan, X.; Song, Y. October( 2007) "Improvement on LEACH Protocol of Wireless Sensor Network",In Proceedings of International Conference on Sensor Technologies and Applications, Valencia, Spain, 14-20, pp. 260-264.

[11] V. Loscrì, G. Morabito and S. Marano. (sept 2005) "A Two-Levels Hierarchy for Low-Energy Adaptive Clustering Hierarchy",Vehicular Technology Conference, VTC 2005 IEEE 62nd, , vol. 3, pp 1809-1813, DOI 10.1109/VETECF 200515558418.

[12] Mo Xiaoyan, (2006)" Study and Design on Cluster RoutingProtocols of Wireless Sensor Networks", Ph.D. Dissertation. Zhejiang University, Hangzhou, China,

[13] Ossama Younis, Sonia Fahmy,(October-December 2004)"HEED:Ahybrid,Energy Efficient,Distributed Clustering a proach for Ad Hoc Sensor Networks" IEEE transactions on Mobile Computing Vol .3,No.4, pp 366-379.

[14] Vevik Katiyar, Narottan Chand, (March 2011)"'Improvement in LEACH Protocol for large -scale WSNs", Proceedings of (ICETECT), Tamil Nadu, pp1070-1075.

[15] Li-Qing Gue, Yi Xie, Chen-Hui Yang, Zhang-Wei Jing, (11-14 July 2010) "Improvement on LEACH by Combining Adaptive Cluster Head Election and Two-Hop Transmission", Proceedings of the Ninth International Conference on Machine Learning and Cybernetics, Qindao, 